\title{
Ovarian Cancer and Primary Peritoneal Carcinoma pNO TNM Finding v7
}

National Cancer Institute

\section{Source}

National Cancer Institute. Ovarian Cancer and Primary Peritoneal Carcinoma pNO TNM

Finding V7. NCI Thesaurus. Code C89656.

Ovarian cancer or primary peritoneal carcinoma without regional lymph node metastasis.

(from AJCC 7th Ed.) 